\title{
Aus der Entwicklungsgeschichte der Wetterkarte ${ }^{1}$
}

\section{Einleitung}

Wetterkarten als graphisch aufgearbeitete Darstellungen des gegenwärtigen atmosphärischen Zustandes sind dem Zeitungsleser oder Fernsehzuschauer sehr wohl bekannt. Nicht geringem Aktualitätsverlust wie eben auch ein Großteil der übrigen in diesen $\mathrm{Me}$ dien aufgenommenen Informationen - unterliegen die Nachrichten über das Wetter. Dem Umstand, daß kartographische Darstellungen des Wetters binnen 24 Stunden veraltet sind, ist die geringe Beachtung der Wetterkarte zuzuschreiben. In Nachschlagewerken und Lehrbüchern der Kartographie findet die Wetterkarte zumeist nur der Vollständigkeit wegen Berücksichtigung.

Wird nach der Ursache des Umstandes gesucht, weshalb die Wetterkarte wenig Eingang in die Literatur und geringes kartographisches Interesse findet, so dürften etwa die folgenden Gründe angeführt werden:

1. Im allgemeinen sind dem Leser oder Zuschauer nur die veröffentlichten, vereinfacht-schematisierten Wetterkarten, die sog. Publikumskarten, bekannt.

2. Der schnelle Aktualitätsverlust der Darstellung verbunden mit der Auflage der einfachen Reproduzierbarkeit - meist ist die Darstellung nur in Schwarzweiß gehalten - veranlaßt zu einer einfachen graphischen Gestaltung.

3. Die genannte einfache graphische Gestaltung sowie der kartographisch nicht komplexe Inhalt bedürfen weit geringerer graphischer Darstellungsmittel und Fertigkeiten, wie dies in aller Regel für andere Karten der Fall ist.

4. Publikumskarten gehen aus synoptischen Arbeitskarten hervor. Letztere sind reine Arbeitsinstrumente, zu deren Ausfertigung keine Kartographen beigezogen werden. Damit entfallen der Einbezug und das Interesse der Kartographie ebenso wie auch der Einsatz von deren gestalterischen Erfahrung.

Die eben gemachten Ausführungen könnten zur vorschnellen Annahme verleiten, die Beschäftigung mit Wetterkarten sei wenig ertragreich. Vom kartographischen Gesichtspunkt aus betrachtet findet die Wetterkarte in der Tat weniger Berücksichtigung als manches andere Teilgebiet innerhalb der thematischen Karto- graphie. Zu Unrecht. Denn in der disziplinen-historischen Beurteilung nimmt die Wetterkarte für das Verständnis meteorologischer Vorgänge eine sehr wichtige Stellung ein. So wäre beispielsweise ohne die Kartendarstellungen in der Meteorologie der Durchbruch in der Prognostik durch die Bergener Schule (auch als Norweger Schule bekannt) nicht denkbar.

\section{Wissenschaftliche Voraussetzungen}

Der Schritt der Meteorologie zur Wissenschaft einerseits sowie die Verfertigung der uns interessierenden Wetterkarten andererseits setzte technische, wissenschaftliche und methodische Entwicklungen voraus. Spätestens ab dem letzten Viertel des 18. Jahrhunderts standen meteorologische Meßgeräte ${ }^{2}$ zur Verfügung, die quantitative Beobachtungen erlaubten. Ebenso war bis dahin der grundsätzliche Zusammenhang zwischen meteorologischen Erscheinungen und Schwankungen der Meßwerte erkannt worden. Die Entwicklung der Maßeinheiten kam im gleichen Zeitraum zu einem ersten Abschluß. Bis Mitte des 18. Jahrhunderts hatte sich die Einsicht durchgesetzt, da $\beta$ die Meßmethoden und Meßbedingungen zu vereinheitlichen sind. Die Notwendigkeit der großräumigen Beobachtungen und deren Zusammenschau sowie die Forderung nach einem koordinierten Beobachtungsnetz, um die Funktionsweise meteorologischer Erscheinungen verstehen zu können, war Anfang des 18. Jahrhunderts erkannt worden.

Die Umsetzung dieser wissenschaftlichen Voraussetzungen in Form eines internationalen, mit einheitlichen Geräten ausgerüsteten und nach einheitlichen Beobachtungsregeln arbeitenden Beobachtungsnetzes erfolgte erstmals durch die 1780 gegründete Societas Meteorologica Palatina.

\section{Vorläufer der Wetterkartierung}

Einhellig ist die Ansicht in der Wissenschaft der Kartengeschichte darüber, daß die älteste und gleichzei-

Urban Schertenleib, Grabenackerstr. 54, 8404 Winterthur 
tig heutigen Wetterkarten am nächsten kommenden Kartendarstellungen die 1686 von EDMOND HALLEY (1688) entworfene Windkarte ist (u.a.: HELLMANN 1897, 5; ROBINSON 1982, 69). Die Karte in HALLEYs Artikel war dazu gedacht, die Passatwinde richtig zu erklären. Dies sei HALlEY nicht gelungen, so HELLMANN $(1897,5)$, er habe jedoch mit seiner Arbeit die erste genauere Übersicht über die Windverteilung innerhalb der Wendekreise geliefert.

In Analogie zur ersten Isogonendarstellung, die ebenfalls von EDMOND HALLEY (1701) stammt, hatte ALEXANDER VON HUMBOLDT 1817 in der Abhandlung "Sur les lignes isothermes» die Darstellungsweise der

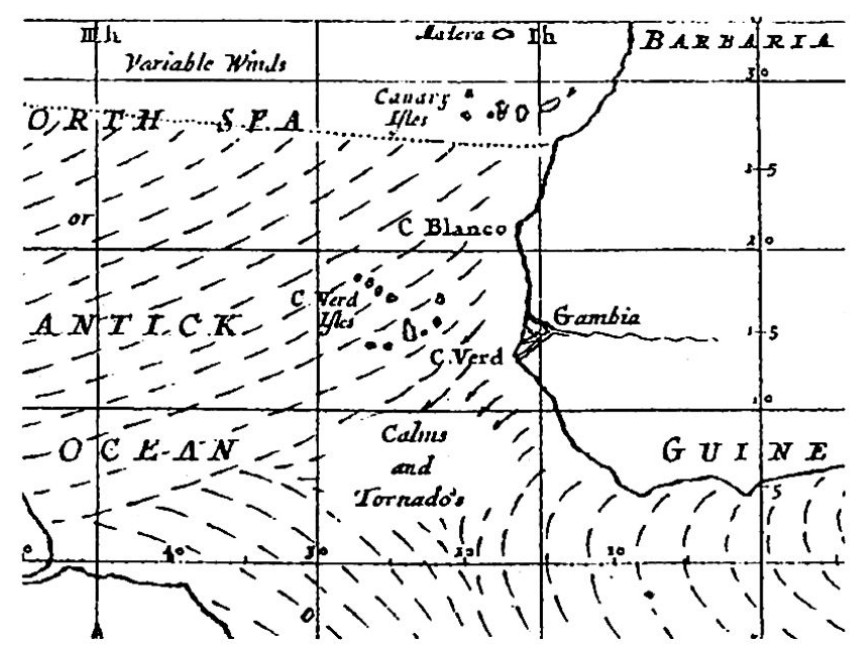

Abb. 1 Ausschnitt aus der Karte von E. HALLEY (1688). Der Strömungssinn des Windes wird durch gestrichelte Linienführung angedeutet; der dickere Teil der Striche zeigt in die Windrichtung. Karte aus HELLMANN (1897). räumlichen Verbreitung mittels Isolinien für die Klimatologie verwendet: HUMBOLDTs Arbeit war ein Meilenstein in der kartographischen Darstellung, weil sie die Wiedereinführung des Gebrauchs der Isolinie in der modernen Wissenschaft nach sich zog.

Obwohl die Darstellung weder Kontinentalumrisse noch sonstige geographische Orientierungspunkte aufweist und als Plattkarte ihren diagrammartigen Charakter betont, wurde sie schnell als Vorbild für kartographische Verbreitungsdarstellungen für Werte mit stetiger Verteilung aufgegriffen. ROBINSON \& WALLIS $(1967,122)$ führen als Beleg für die Bedeutung der an sich schon seit über 200 Jahren bekannten Isoliniendarstellung an, daß die Karte von HUMBOLDT im 3. Band in Gehlers Physikalischem Wörterbuch (1827) reproduziert wurde und weiterentwickelte Anwendungen durch L.F. KAEMTZ (1832) und J. FORBES (1836) Eingang fanden. Schon 20 Jahre nach HUMBOLDTs Karte ist es fast undenkbar, daß der Physikalische Atlas von HEINRICH BERG HAUS (1838) - der erste thematische Atlas überhaupt - noch ohne eine Isolinie auskommen könnte.

Sieht man sich die zwei bis jetzt vorgestellten Karten unter dem Gesichtswinkel genauer an, ob es sich bei ihnen wirklich schon um Wetterkarten im eigentlichen Sinne handelt, so müssen sie dem Inhalt nach den Klimakarten zugewiesen werden. Diese "veranschaulichen die regionale Vielfalt atmosphärischer $\mathrm{Zu}$ stände und Witterungsabläufe aufgrund langjähriger Beobachtungen» eher als Wetterkarten, die den Leser über den uaugenblicklich herrschenden meteorologischen Zustand und über die Wettervorhersage" (WILHELMY 1981, 278) unterrichten. Die Möglichkeit je-

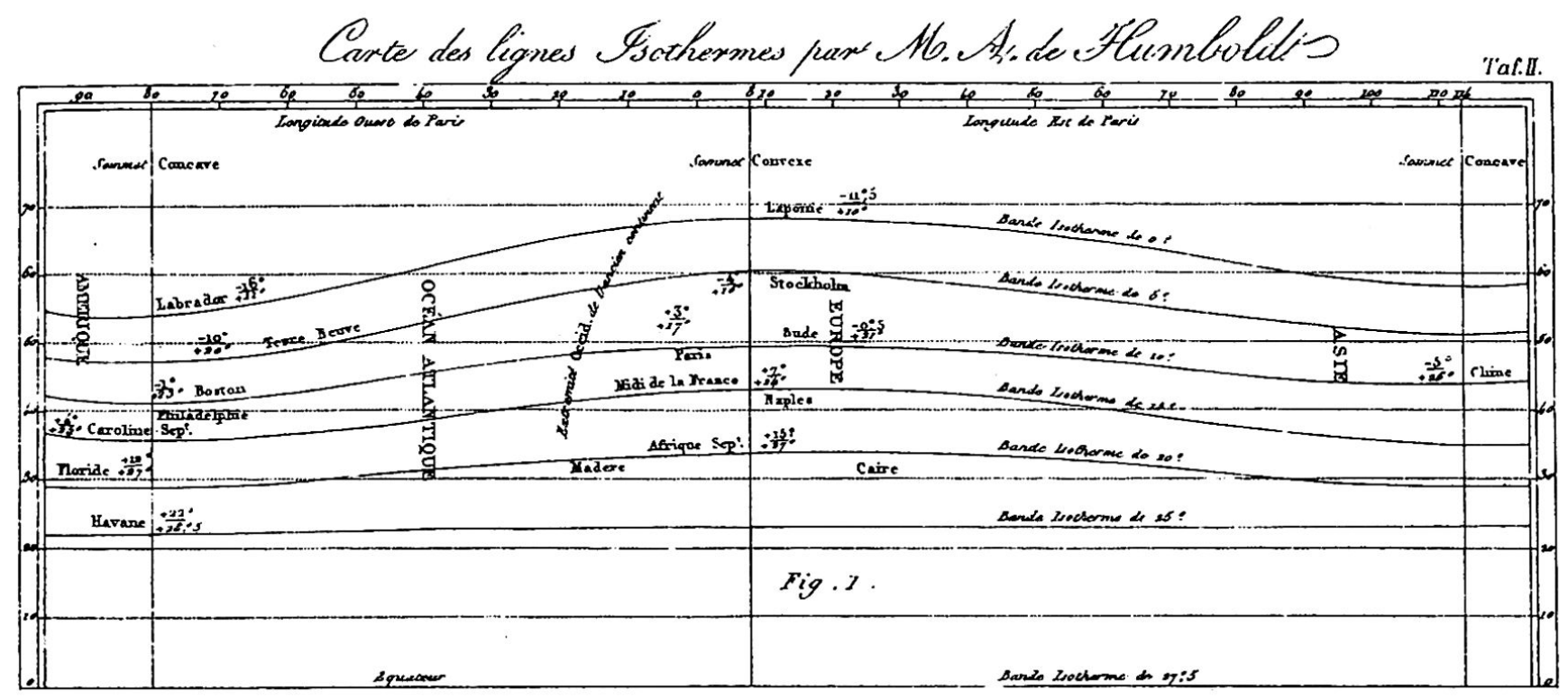

Abb. 2 Carte des lignes Isothermes von A. V. HUMBOLDT (1817). 
METEOROLOGICAL TABLE, Showing the State of the Weather at enob of tho following places at Nino o'clock yenterday morning.

\begin{tabular}{|c|c|c|c|}
\hline Namo of Place. & $\begin{array}{l}\text { Course of } \\
\text { Wind. }\end{array}$ & $\begin{array}{l}\text { 8trongth of } \\
\text { wlind. }\end{array}$ & State of Veather. \\
\hline 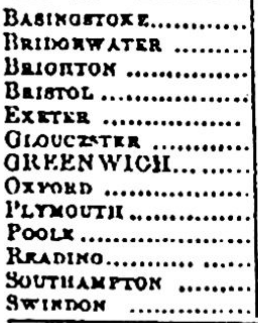 & $\begin{array}{l}\text { w. } \\
\text { N.w. } \\
\text { N. } \\
\text { N.w. } \\
\text { N.w. } \\
\text { N.P. } \\
\text { N.E. } \\
\text { N.w. } \\
\text { 8.w. } \\
\text { N. } \\
\text { N.w. } \\
\text { N.E. } \\
\text { N. }\end{array}$ & $\begin{array}{l}\text { Gentle Breezo } \\
\text { Oentlo Breezo } \\
\text { Gentlo Breezo } \\
\text { Oentlo Breeze } \\
\text { Gentle Breezo } \\
\text { Gentlo Breczo } \\
\text { Oentlo Breezo } \\
\text { Calm } \\
\text { Gentlo Brerzo } \\
\text { Gentlo Breeze } \\
\text { Gentlo Breezo } \\
\text { Calm } \\
\text { Oontlo Breezo }\end{array}$ & 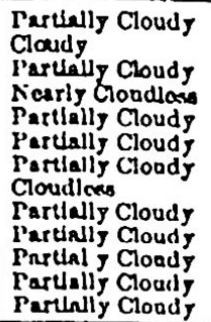 \\
\hline
\end{tabular}

As explainod olsewhere, the rery extensire arrangements neccesary for making this tablo completo haro not jot been concluded. In a abort time wo shall present daily retaras in addition to those abore giren, from Berwick, Birmingham, Bieckburne, Cambridge, Carlisle, Colchester, Conway, Crewe, Darlington, Dundee, Kdinbargh, Folkentone, Galway, Halifax, Hartlepool, Hastings, Holyhead, Kingston, Lanark, Lancester, - Leedo, Leighton, Limcrick. Lirerpool, Manchester, Moffat, Nowcestlo, Peterborongh, Portsmoath, Ilochdale, Rngby, Shap, Sheffield, Sundorlend, Tamworth, Tunbridge, Whitby, and York.

Abb. 3 Erster telegraphischer Wetterbericht in Daily News, London, 14.7.1849.

doch, Echtzeit-Wetterkarten zu zeichnen, ergab sich erst mit der Entwicklung und Einführung leistungsfähiger Fernmeldemittel. Ab dem 14. Juni 1849 wurden erstmals in einer Zeitung überhaupt in Daily News, London, telegraphische Wetterberichte veröffentlicht.

Für die Dauer der Weltausstellung vom 8.8.-11.10.1851 in London demonstrierte die Postverwaltung die Leistungsfähigkeit ihrer telegraphischen Dienste durch die Herausgabe von täglich erschienenen synoptischen Wetterkarten (SYMONS 1896). Nach dieser versuchsweisen Herausgabe von Wetterkarten wurde das Erscheinen wieder eingestellt. Erst ab dem 16.9.1863 erschienen unterbruchslos täglich Wetterkarten in Paris, herausgegeben vom Bureau Central Météorologique (HARRINGTON 1896, Tafel I).

Bevor die Telegraphie erfunden worden war, erstreckte sich die kartographische Darstellung des Wetters auf die nachträgliche graphische Umsetzung von Meßdaten. Alle uns bekannten Wetterkarten, die vor 1851 gezeichnet und veröffentlicht wurden, sind weder zeitgerechte Darstellungen des atmosphärischen Zustandes noch einer allfälligen Prognose. So liegen beispielsweise zwischen den Messungen und der Veröffentlichung der ersten Wetterkarten überhaupt fast fünf Jahre: HEINRICH WILHELM BRANDES legte seiner 1826 publizierten Dissertation Beobachtungen von Weihnachten 1821 zugrunde.
Die Karte überrascht durch die fehlenden Orientierungspunkte wie Küstenverlauf, Flüsse oder Gebirgszüge. Die Beobachtungsorte sind ihrer ungefähren Lage entsprechend eingezeichnet. Der Nullmeridian geht in dieser Darstellung - wie damals üblich - durch die Kanareninsel Ferro. Die Zahlenangaben in der Abbildung geben die negative Abweichung vom mittleren Luftdruck des Beobachtungsortes in Pariser Linien $^{3}$ an (sCULTETUS 1943, 358). Bei genauerem Vergleich der vier Karten, die BRANDES' Ausführungen beigelegt sind, läßt sich die Ostwärtswanderung des Tiefdruckgebietes nachvollziehen.

Neben dieser Darstellung von BRANDES existiert eine Wetterkartenrekonstruktion von HILDEBRANDSSON \& TEISSERENC DE BORT (1899), die aufgrund einer Textauslegung von BRANDES'Beiträge zur Witterungskunde (1820) angefertigt wurde.

BRANDES hatte nämlich in einem Beitrag in Gilberts Annalen der Physik und Chemie (61 [1819] 113) die Idee geäußert, daß farbige Darstellungen in Kärtchenform, welche die Verteilung des blauen Himmels oder wol-

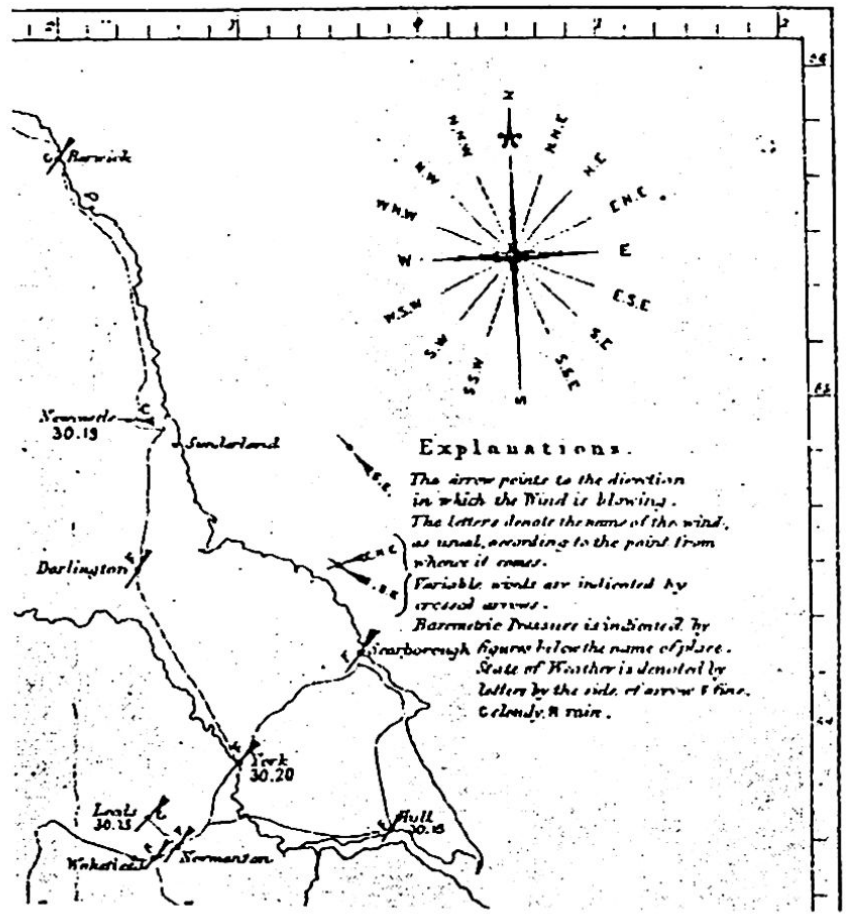

Abb. 4 G.J.SYMONS (1896): Erste tägliche Wetterkarte, vertrieben anläßlich der Weltausstellung von 1851 in London (Ausschnitt).

Nebst einer Windrose erklärt eine Legende die Darstellung: EXPLANATIONS: The arrow points to the directions in which the Wind is blowing. The letters denote the name of the wind, as usual, according to the point from whence it comes./Variable winds are indicated by crossed arrows./Barometric pressure is indicated by figures below the name of place./ State of Weather is denoted by the side of arrow F fine, $C$ cloudy, $R$ rain.

Druckangaben in Inch und 100 Teilen davon. 


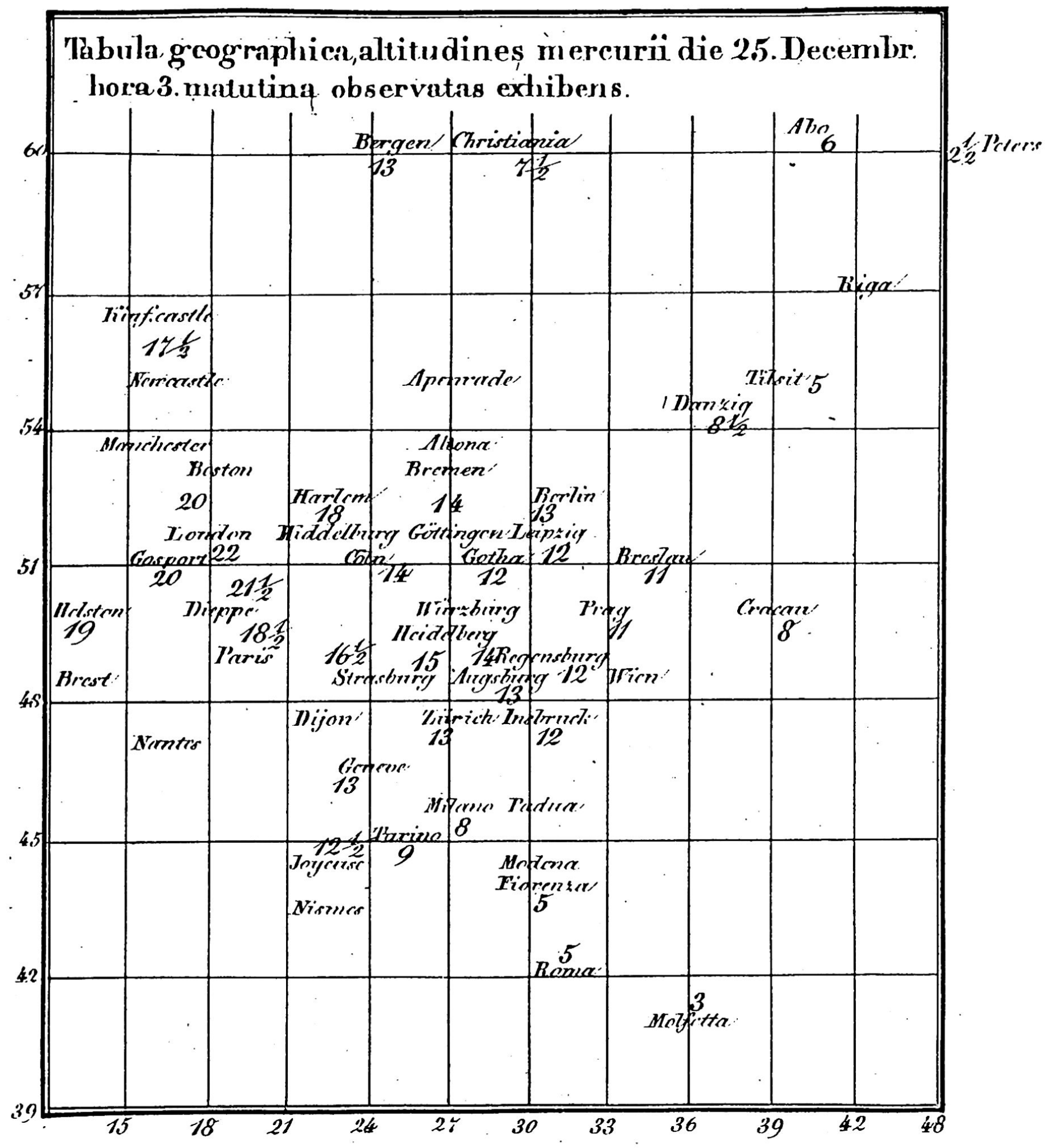

Abb. 5 H.W. BRANDES (1826): Wetterkarte vom 25.12.1821, 03.00 Uhr. 


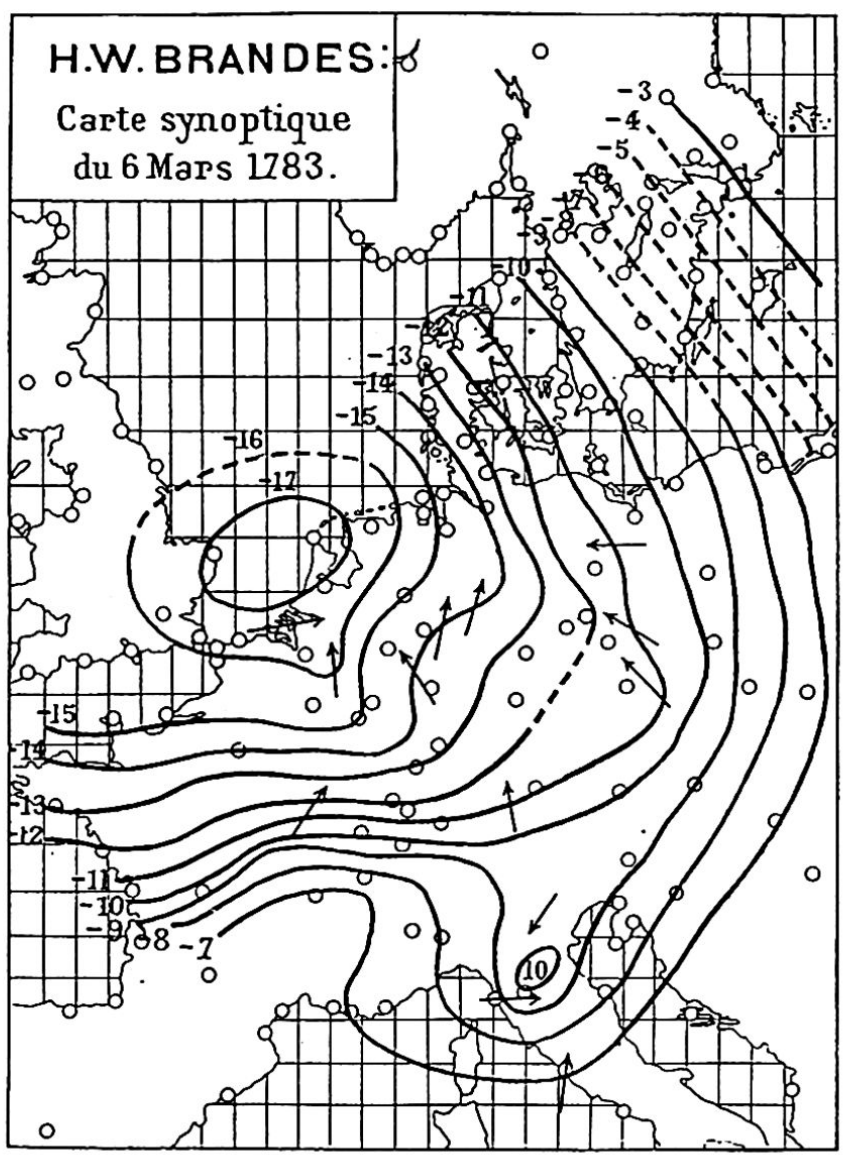

Abb. 6 Kartenrekonstruktion von HILDEBRANDSSON \& TEISSERENC DE BORT (1899). Die Beobachtungswerte in dieser Karte stammen aus den Ephemeriden der Mannheimer Societas Meteorologica Palatina.

ken- und regenverhangener Gebiete zeigten und mit Pfeilen an den Stellen der Beobachtung die Windrichtung angäben, "dem Publikum mehr Vergnügen und Belehrung gewähren würden als Witterungstafeln». An anderer Stelle im Text (S. 421-422) bedauerte er, daß er mangels vollständiger Barometerbeobachtungen nicht die Linien gleicher Druckabweichung als verzerrte Kreise um ein Zentrum habe erhalten können. Die eben angeführten Linien gleicher Druckabweichung bezog BRANDES auf die nicht reduzierten Mittelwerte des Druckes der Beobachtungsorte.

Die in den Textstellen erwähnten kartographischen Elemente Pfeil und (Iso-)Linie wurden erst von HILDEBRANDSSON \& TEISSERENC DE BORT $(1899,47)$ in die Rekonstruktion eingebracht. Interessanterweise hatte BRANDES die Methode der oben erwähnten verzerrten Kreise zur Darstellung der Druckverteilung in seiner Dissertation (vgl. Abb. 5) nicht eingesetzt, wo sie der Darstellung zu bedeutend mehr Übersicht und Ausdruck verholfen hätte. Es scheint, daß BRANDES den ungefähr kreisförmigen Verlauf ähnlicher mittlerer Druckabweichungen um das Zentrum eines Tiefdruckgebietes zwar gedanklich erkannt, seine $\mathrm{Er}$ - kenntnisse aber nicht graphisch zu Papier gebracht hatte. Für die Behauptung von HILDEBRANDSSON \& TEISSERENC DE BORT, BRANDES habe schon vor 1826 Wetterkarten gezeichnet, gibt es jedenfalls keine schlüssigen Hinweise.

Neben den Forschungen in Europa wurden in den Vereinigten Staaten im Zeitraum von 1830 bis 1850 große Anstrengungen zur Untersuchung von Stürmen (Tornados und Hurrikane) unternommen. Mit Darstellungen traten ELIAS LOOMIS (1811-1889), JAMES POLLARD ESPY (1785-1860) und WILLIAM C. REDFIELD (1789-1857) hervor.

REDFIELD beschäftigte sich mit Wirbelstürmen und Wirbelformen, der Fließrichtung der einströmenden Luft sowie Zugbahn der Stürme. Er erkannte, daß die Luft tangential in Wirbelform und nicht von allen Seiten zugleich (zentripetal) ins Tief fließt. Die Darstellung (Abb.7) zeigt die beobachteten Windrichtungen. Durch die konzentrischen Kreise, die um das angenommene Zentrum für den Mittag vom 15.12.1839 eingezeichnet sind, wird die wirbelförmige Anordnung der Pfeile hervorgehoben. Die Ziffern neben den Pfeilen geben die Nummern der einzelnen Stationen an. Entlang der gestrichelten, diagonal verlaufenden Linie nahm REDFIELD die Zugbahn der Zyklone an.

Um die Vielfalt der Abbildungen aufzuzeigen, ließen sich die bis hier vorgestellten Karten um mehr als ein Dutzend zusätzlicher Darstellungen erweitern. Aus den Anfangszeiten stammen auch farbige Wetterkarten, die in ihrer Art selbständig sind und sich nicht durch die Farbigkeit allein, sondern auch durch die Darstellung an sich hervorheben. Ab Mitte der 1860er Jahre erfahren die Wetterkarten inhaltlich keine Bereicherung mehr und entsprechen jener des Wetterberichtes der Schweizerischen Meteorologischen Centralanstalt in Zürich vom 5.10 .1880 (Abb. 8).

Die Synoptik war ab den 1860er Jahren bis 1918/1919 durch die Interpretation der Isobaren geprägt. In diese beiden Jahre fiel die Entwicklung der Frontentheorie durch die BergenerSchule. Die neuen Erkenntnisse führten zu einem Durchbruch im Verständnis zykonaler Prozesse sowie in der Wetterprognose. Mit der Ankündigung eines neugestalteten Wetterberichtes für den 1.1.1946 zog die heute übliche Frontendarstellung in den veröffentlichten Wetterkarten der Schweizerischen Meteorologischen Anstalt ein.

\section{Anmerkungen}

${ }^{1}$ Der Artikel basiert auf Auszügen der Diplomarbeit Die Wetterkarte bis zur Einführung der Frontentheorie 1918/1919 Ein Beitrag zur Entwicklungsgeschichte der Thematischen Kartographie (1989) des Autors, ausgeführt am Geographischen Institut der Universität Zürich.

${ }^{2}$ Für eine Einführung in die Instrumentengeschichte vgl. u.a. MIDDLETON (1969) oder die Bibliographie von BRUSH \& LANDSBERG $(1985,327-334)$.

${ }^{3} 1$ Pariser Linie $=2,26 \mathrm{~mm}$ Quecksilbersäule $\cong 3 \mathrm{mb}$ 


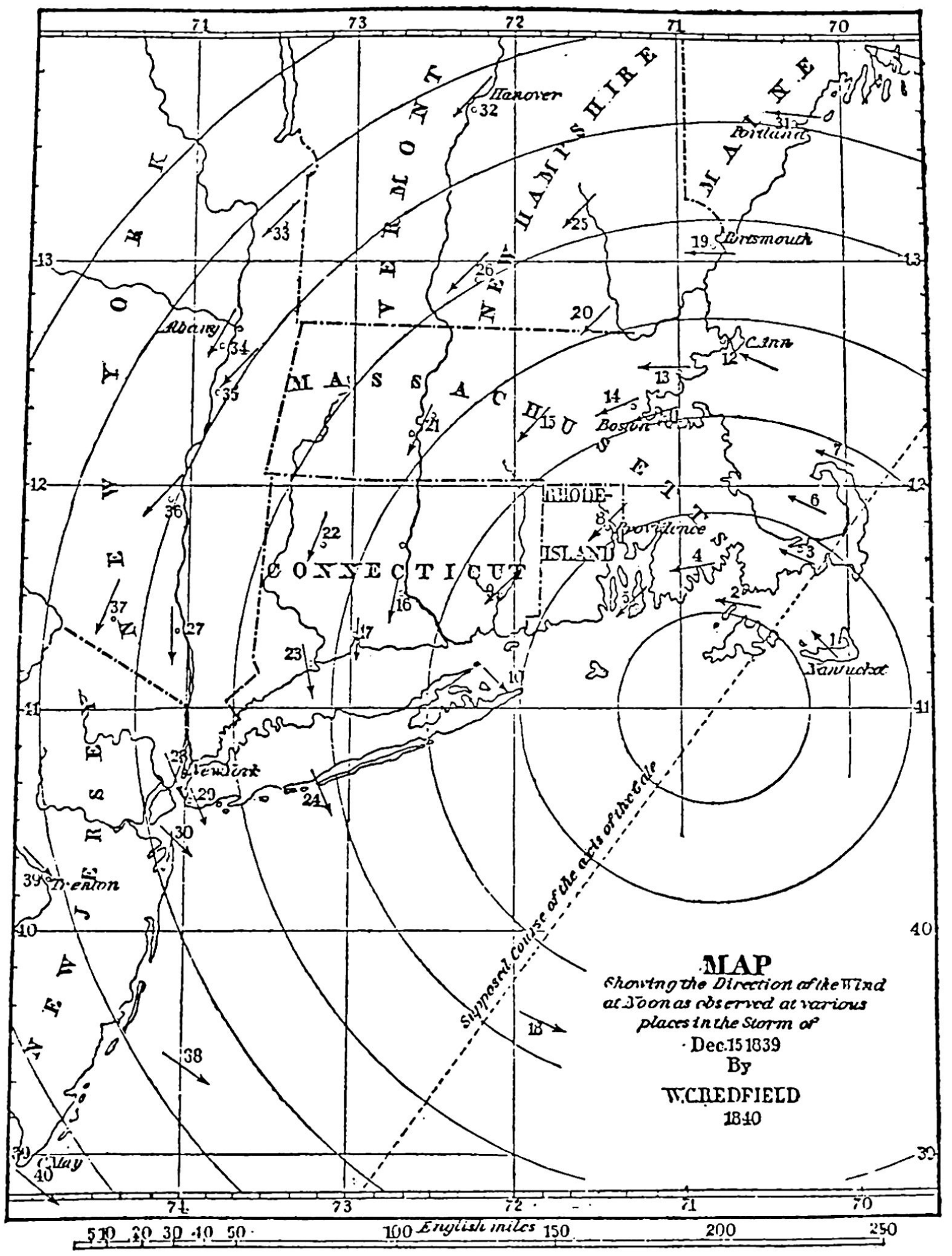

Abb. 7 W.C. REDFIELD (1843), Map showing the Direction of the Wind at Noon as observed at various places in the Storm of Dec. 15. 1839. 


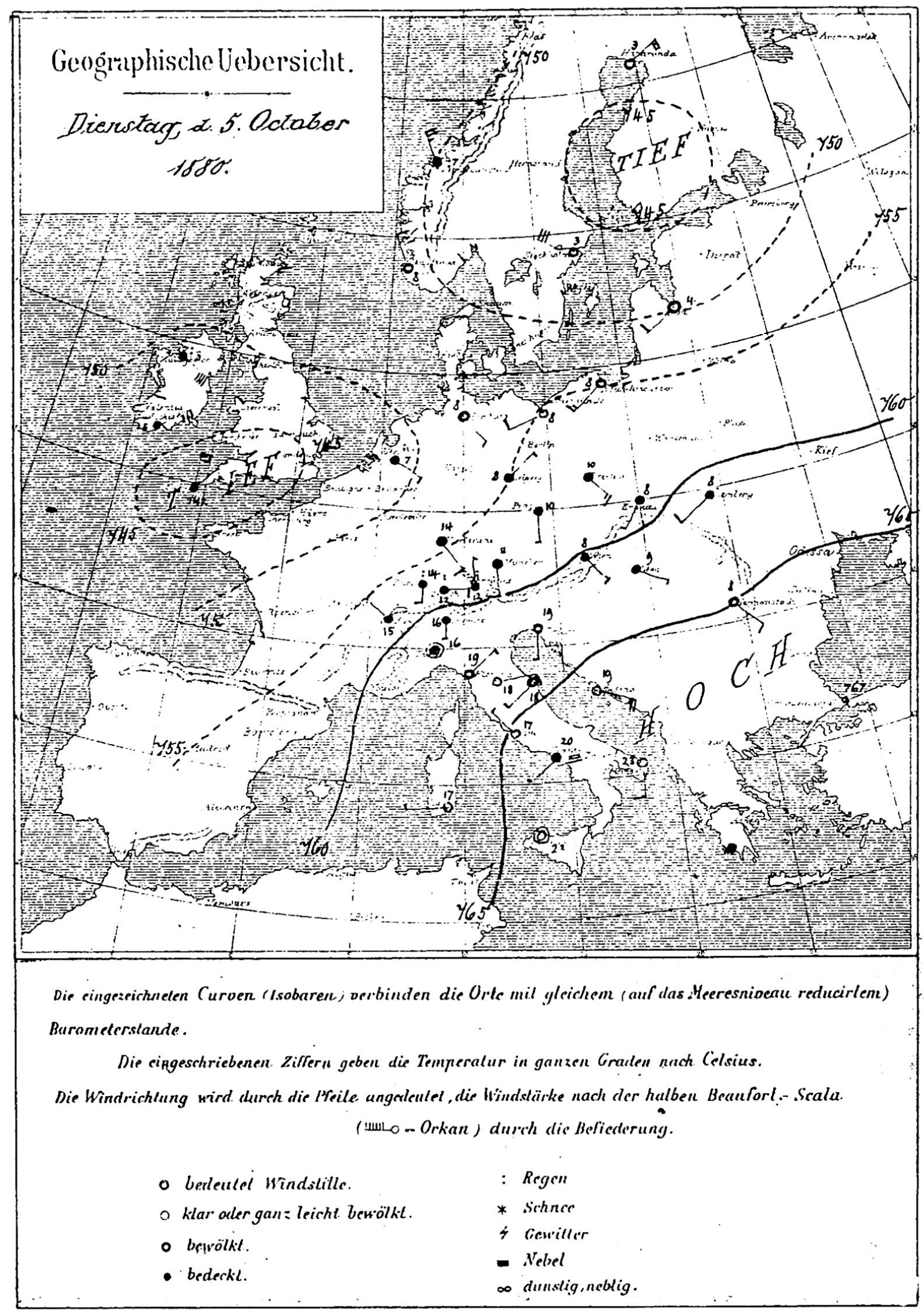

Abb. 8 Wetterkarte der Schweizerischen Meteorologischen Centralanstalt in Zürich vom 5.10.1880. 


\section{Literatur}

BERGHAUS, H. (1838): Dr. Heinrich Berghaus'Physikalischer Atlas oder Sammlung von Karten, auf denen die hauptsächlichsten Erscheinungen der anorganischen und organischen Natur nach ihrer geographischen Verbreitung und Verteilung dargestellt sind, Gotha.

BRANDES, H.W. (1820): Beiträge zurWitterungskunde, Untersuchungen über den mittleren Gang der Wärme-Änderungen durchs ganze Jahr; über gleichzeitige Witterungs-Ereignisse in weit voneinander entfernten Weltgegenden ..., Leipzig.

BRANDES, H.W. (1826): Dissertatio physica de repentinis variationibus in pressione atmosphaerae observatis, Lipsiae (Leipzig)

BRUSH, S.G. \& H.E. LANDSBERG (1985): The History of Geophysics and Meteorology, An Annotated Bibliography. In: Bibliographies of the History of Science and Technology, Vol. 7, New York/London.

ESPY, J.P. (1841): The Philosophy of Storms.

FORBES,J. (1836) (übers. von W. Mahlmann): Abriß einer Geschichte der neueren Fortschritte und des gegenwärtigen Zustandes der Meteorologie, Berlin.

HALLEY, E. (1688): An historical Account of the Trade Winds, and Monsoons, observable in the Seas between and near the Tropics, with an attempt to assign the Phisical cause of the said Winds. In: Philosophical Transactions of the Royal Society, No. 183 (1688) 153-168, London.

HALLEY, E. (1701): Tabula Nautica, London. Reprint in HELLMANN (1895).

HARRINGTON, M.W. (1895): History of the Wheather Map. In: Report of the international meteorological Congress, Chicago III., Aug. 21-24, 1893, Part II, pp. 326-335, Washington.

HELLMANN, G. (1895) (Hrsg.): Die ältesten Karten der Isogonen, Isoklinen, Isodynamen. In: Neudrucke von Schriften und Karten über Meteorologie und Erdmagnetismus No. 4, Berlin.

HELLMANN, G. (1897) (Hrsg.): Meteorologische Karten. In: Neudrucke von Schriften und Karten über Meteorologie und Erdmagnetismus No. 8, Berlin.
HILDEBRANDSSON, H.H. \& DE L. TEISSERENC DE BORT (1898-1907): Les Bases de la Météorologie Dynamique, Historique Etat des nos Connaissances (I-VII), Paris.

HUMBOLDT, A., VON (1817): Sur les lignes istothermes, par A. de Humboldt (Extrait). In:Annales de chimie et de physique, 5 (1817) 102-111, Karte zwischen Seite 112 und 113, Paris. Literaturangabe zit. nach ROBINSON \&WALLIS $(1967,122)$.

KAEMTZ, L.F. (1832-1836): Lehrbuch der Meteorologie, 3 Bände, Leipzig.

KUTZBACH, G. (1979): The Thermal History of Cyclones, A History of Meteorological Thought in the Nineteenth Century, Boston.

LOOMIS, E. (1841): On the Storm which was experienced throughout the United States about the 20th of December, 1836. In: Transactions of the American Philosophical Society, 7 (1841) 125-163 mit Tafel.

LOOMIS, E. (1846): On the Storm which was experienced throughout the United States of America, in the month of February, 1842. In: Transactions of the American Philosophical Society, 9 (1846) 161-184 mit Tafel.

MIDDLETON, W.E.K. (1969): Invention of the Meteorological Instruments, Baltimore.

REDFIELD, WILLIAM C. (1843): Observations on the storm of December 15, 1839. In: Transactions of the American Philosophical Society, 8 (1843) 77-80.

ROBINSON, A.H. \& H.M. WALLIS (1967): Humboldt's Map of Isothermal Lines: A Milestone in Thematic Cartography. In: The Cartographic Journal, 4 (1967) 119-123.

ROBINSON, A.H. (1982): Early Thematic Mapping in the History of Cartography, Chicago/London.

SCULTETUS, H.R. (1943): Die erste Wetterkarte. In: Meteorologische Zeitschrift, $60(1943,10)$ 356-359.

SCHWEIZERISCHE METEOROLOGISCHE CENTRALANSTALT (1880): Wetterberichte der Schweizerischen Meteorologischen Centralanstalt in Zürich, vom 5.10.1880.

SYMONS, G.J. (1896): Scientific Wheather Forecasting. In: Science Progress N.S., 1 (1896) 1-12.

WILHELMY, H. (1981): Kartographie in Stichworten, Hirt's Stichwörterbücher, in 3 Teilen, 4., überarbeitete Auflage, Kiel. 